
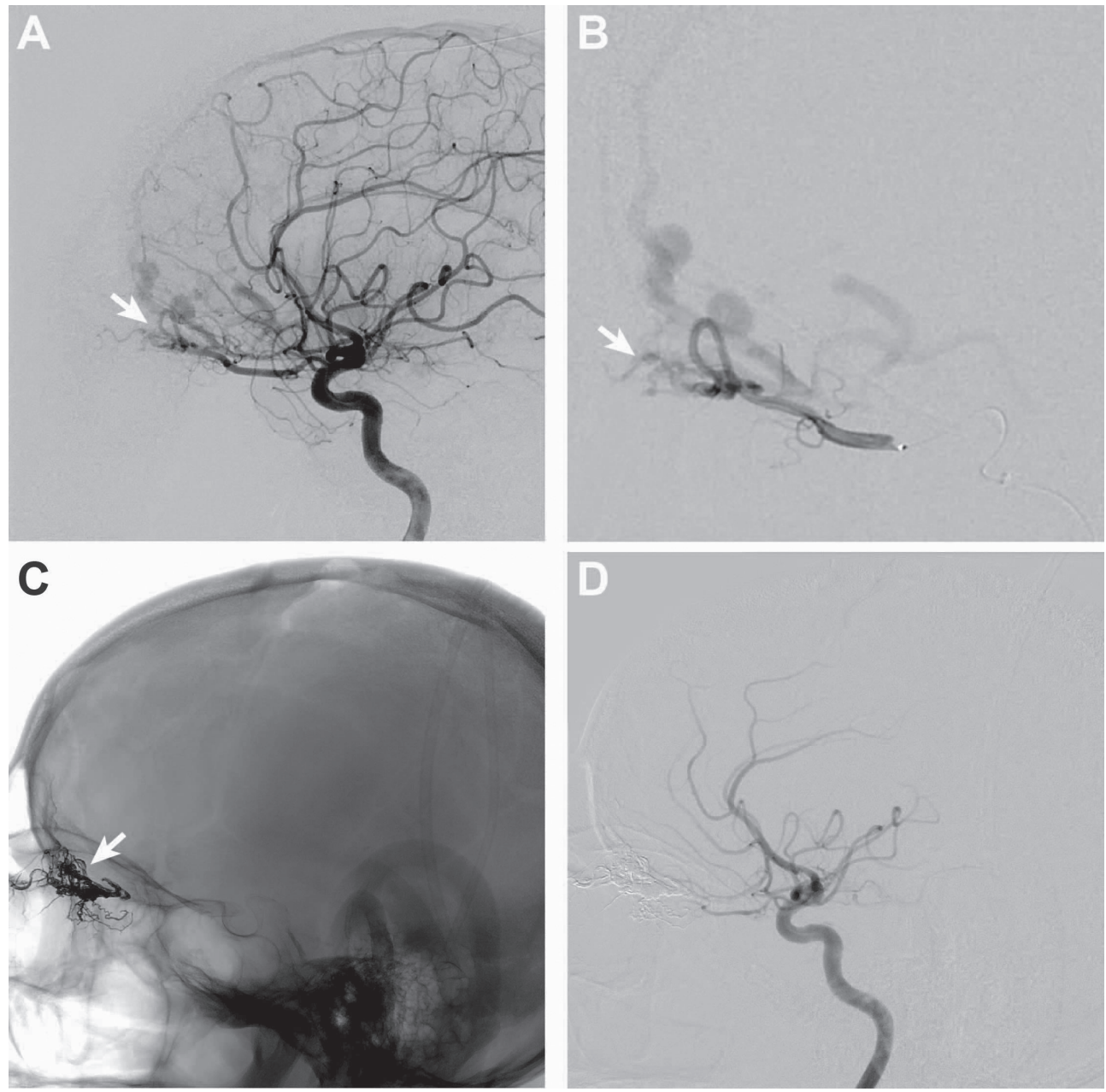

Abstract E-008 Figure 1

\section{E-009 A NOVEL TRANSVENOUS APPROACH TO EMBOLIZATION OF AN EXTENSIVE TRANSVERSE AND SIGMOID SINUS DAVM IN A PATIENT WITH AN OCCLUDED IPSILATERAL INTERNAL JUGULAR VEIN}

M Adix, II, I Choi, I Kaminsky. Interventional Neuroradiology, Lahey Hospital and Medical Center, Burlington, MA

\subsection{6/neurintsurg-2016-012589.81}

For patients in whom treatment is warranted, dural arteriovenous malformations (DAVMs) can be managed via open surgery, endovascular embolization, or radiation therapy. Endovascular management can be performed from either the arterial or venous side. We describe the case of a patient who presented with left-sided pulsatile tinnitus. Initial cerebral angiogram demonstrated an extensive DAVM of the left transverse and sigmoid sinuses being supplied primarily by branches of the left internal carotid, middle meningeal, and occipital arteries and the right occipital artery. We also observed cortical venous reflux compatible with venous hypertension. Given the extensive arterial supply, a transvenous approach to treatment was chosen. Upon attempted catheterization of the left internal jugular vein (IJ), we discovered that it was occluded up to the level of its junction with the left facial vein. The occluded segment could not be catheterized, so we accessed the patent superior portion of the left IJ via a circuitous route through the left paravertebral plexus. Once the left transverse sinus was successfully catheterized, two major vascular pedicles draining into the transverse sinus and transverse-sigmoid junction were closed with detachable coils in a sinus-sparing procedure. Final angiography demonstrated restoration of anterograde flow through the left transverse and sigmoid sinuses with resolution of the previously seen cortical venous reflux. Only a small residual vascular shunt into the left sigmoid sinus remained. This case demonstrates that patients with DAVMs and ipsilateral IJ obstruction can still be successfully treated via a transvenous approach using alternative venous channels.

Disclosures M. Adix: None. I. Choi: None. I. Kaminsky: None.

\section{E-010 VESSEL LENGTH SPLINE MEASUREMENT WITH ECHOPIXEL TRUE 3D VIEWER}

I Kaminsky, M Adix, I Choi. Interventional Neuroradiology, Lahey Hospital and Medical Center, Burlington, MA

\subsection{6/neurintsurg-2016-012589.82}

Introduction/purpose Determining the appropriate device length for flow diverter placement or stent assisted coiling can be limited by inaccurate vessel length spline measurement on typical 3D workstations especially in the setting of marked vessel tortuosity, overlying structures, and large aneurysm size. 


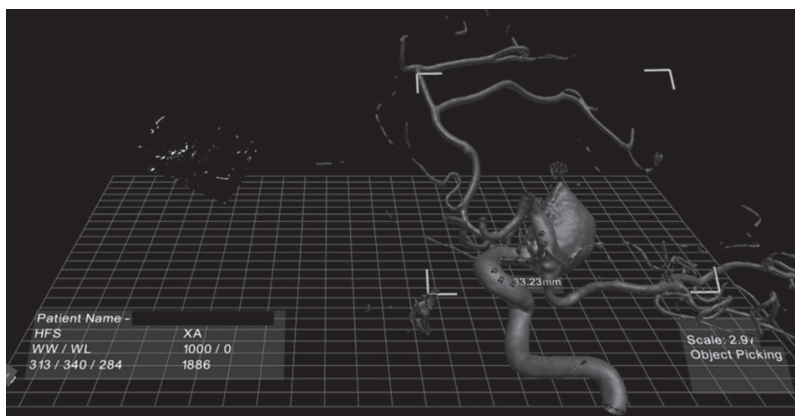

Abstract E-010 Figure 1

Echopixel software allows the user to more accurately select the intended structure of interest for measurement with true $3 \mathrm{D}$ visualization and active feedback. Early experience with the Echopixel True 3D Viewer appears to provide more reliable vessel length measurements in this setting.

Materials and methods Two separate patient data sets were utilized to measure the internal carotid artery length from the normal vessel beyond an intracranial aneurysm to the horizontal cavernous segment on both the Siemens Leonardo 3D Workstation and the Echopixel True 3D Viewer utilizing the spline function. Measurements were repeated 10 times on each workstation for each data set. The first patient data set included measurements from the anterior choroidal artery level to the proximal horizontal cavernous segment of the internal carotid artery with limited underlying tortuosity and a small aneurysm. The second patient data set included measurements from just distal to the anterior choroidal artery origin to the mid horizontal cavernous segment of the internal carotid artery with marked underlying tortuosity, overlapping vascular structures and a large adjacent aneurysm.

Results Measurements from the first patient data set demonstrated a mean of $31.0 \mathrm{~mm}$ on Echopixel with a range of $30.68 \mathrm{~mm}$ to $31.62 \mathrm{~mm}$. The mean on Leonardo was 30.9 $\mathrm{mm}$ with a range of $30.0 \mathrm{~mm}$ to $33.4 \mathrm{~mm}$. Measurements on the second patient data set demonstrated a mean of $33.7 \mathrm{~mm}$ on Echopixel with a range of $32.5 \mathrm{~mm}$ to $34.5 \mathrm{~mm}$. The mean on Leonardo was $44.9 \mathrm{~mm}$ with a range of $35.0 \mathrm{~mm}$ to $101.3 \mathrm{~mm}$.

Conclusion Both the measurements taken on the Echopixel True 3D Viewer and the Siemens Leonardo 3D Workstation were reproducible with excellent accuracy when evaluating an internal carotid artery with limited tortuosity, minimal overlying structures and a small aneurysm. When marked tortuosity, overlying vascular structures, and larger aneurysms are present, the Leonardo workstation will occasionally select unintended structures as the point to be measured, resulting in markedly inaccurate assessment of vessel length. Echopixel allows the user to view the precise points where the measurements are being placed in 3D with on screen and haptic feedback. This improved reproducibility/accuracy may be beneficial in selecting an appropriately sized device.

Disclosures I. Kaminsky: None. M. Adix: None. I. Choi: 2; C; Codman.

\section{E-011 SOFIA DISTAL ACCESS CATHETER FOR ENDOVASCULAR TREATMENT OF ACUTE ISCHEMIC STROKE USING COMBINED MECHANICAL AND ASPIRATION THROMBECTOMY}

1J Wong, ${ }^{1} \mathrm{H}$ Do, ${ }^{1} \mathrm{~N}$ Telischak, ${ }^{1} \mathrm{~A}$ Moraff, ${ }^{1} \mathrm{M}$ Marks, ${ }^{2} \mathrm{R}$ Dodd, ${ }^{1} \mathrm{~J}$ Heit. 'Interventional Neuroradiology, Stanford University, Stanford, $C A ;{ }^{2}$ Neurosurgery and Interventional Neuroradiology, Stanford University, Stanford, CA

\subsection{6/neurintsurg-2016-012589.83}

Introduction Mechanical thrombectomy with stent retrievers is superior to medical management in acute ischemic stroke due to large vessel occlusion (LVO). The Direct Aspiration First Pass (ADAPT) and combined mechanical/aspiration thrombectomy (Solumbra) techniques are used in endovascular stroke treatment; these techniques require an intermediate catheter for suction at the clot interface. SOFIA (Soft torqueable catheter Optimized For Intracranial Access) is a single lumen flexible catheter with coil and braid reinforcement developed for intracranial use. We describe our initial experience with SOFIA in acute stroke intervention and evaluate its efficacy and safety.

Methods Our institutional review board approved this study. We retrospectively identified all patients undergoing endovascular stroke therapy using SOFIA from our database. Patient demographic data, stroke presentation, treatment details, and complications were recorded from the medical record. The primary outcome was successful revascularization (TICI IIb or III) and the number of passes for revascularization. Secondary outcome measures were complication rates and NIHSS score on discharge.

Results 33 patients (20 females, 13 males) were treated for LVO using the SOFIA catheter. Mean patient age was 71.9 years. Intravenous tPA was administered in 22 patients (67\%). Vessel occlusion predominantly affected the anterior circulation (10 left sided and 21 right sided) and involved the ICA terminus (5 patients, 15.2\%), M1 (16 patients, 48.5\%) and M2 (8 patients, 24.2\%) segments. 4 patients (12.1\%) had posterior circulation strokes. Mean NIHSS was 14.2 (95\% CI 12.316.2) on presentation and 7.8 (95\% CI 4.9-10.6) on discharge. The Solumbra technique was used in 31 patients (94\%), ADAPT in one patient (3\%), and intra-arterial
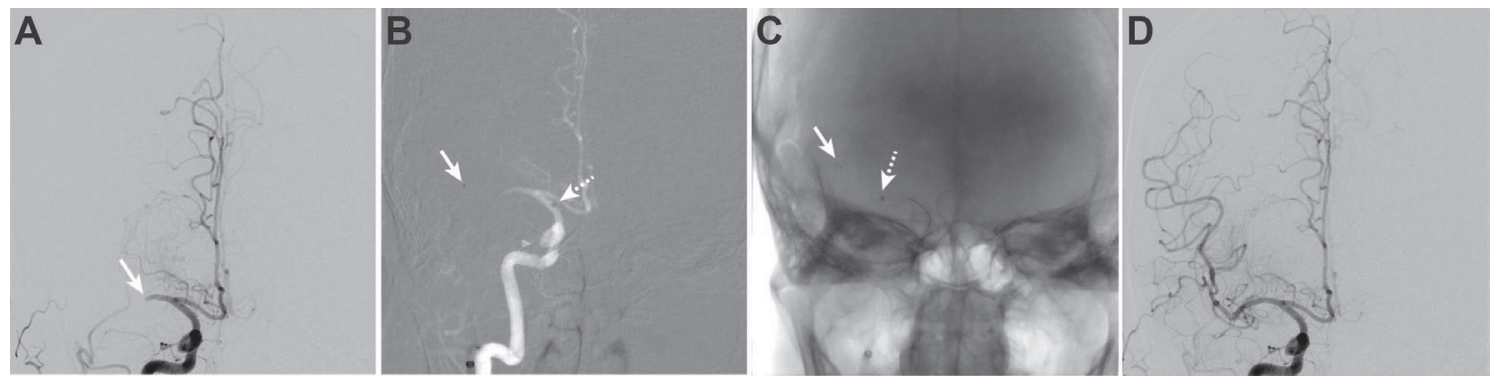